OPEN ACCESS

Edited by:

Saleh Abbas,

Deakin University, Australia

Reviewed by:

Fabrizio Romano,

University of Milano-Bicocca, Italy

Jose M. Ramia

Servicio de Salud de Castilla La

Mancha, Spain

*Correspondence:

Fang Liu

sxliuf77@163.com

Specialty section:

This article was submitted to

Visceral Surgery,

a section of the journal

Frontiers in Surgery

Received: 12 October 2020 Accepted: 29 December 2020

Published: 01 February 2021

Citation:

Shao H, Lu B, Shen Z and Liu F (2021)

Sclerosing Angiomatoid Nodular

Transformation of the Spleen: Analysis

of Clinical and Pathological Features in

Five Cases. Front. Surg. 7:609284.

doi: 10.3389/fsurg.2020.609284

\section{Sclerosing Angiomatoid Nodular Transformation of the Spleen: Analysis of Clinical and Pathological Features in Five Cases}

\author{
Huijiang Shao, Baochun Lu, Zhihong Shen and Fang Liu* \\ Department of Hepatobiliary and Pancreatic Surgery, Department of Pathology, Shaoxing People's Hospital, Shaoxing \\ Hospital of Zhejiang University, Shaoxing, China
}

Objective: We aimed to summarize the clinical and pathological features of sclerosing angiomatoid nodular transformation (SANT) in spleen among five cases.

Methods: Five cases (male: 3; female: 2; mean age: 47.6 years) with SANT confirmed by pathological analysis between July 2010 and November 2019 in our hospital were included in this study. The clinical, imaging, and pathological data were analyzed retrospectively.

Results: Three patients presented with mild abdominal pain or discomfort, while the other two were symptom free. Two patients received ultrasonography (US), and all patients underwent a computerized tomography (CT) scan in our hospital. The typical "spoke wheel" pattern was seen in two cases, and central calcification was detected in one case on the CT scans. All patients indicated peripheral enhancement around the SANT lesion during the arterial phase. Open or laparoscopic splenectomy was performed for treatment. No patient showed recurrence in the follow-up. The pathological characteristics of our cases were in line with those of previous literatures.

Conclusions: Peripheral enhancement around the SANT lesion during the arterial phase should be taken into consideration for the diagnosis of SANT as an imaging sign on CT scans. Special attention should be paid to the splenic integrality during the laparoscopic approach, due to the probability of malignancy and the fragility of the spleen.

Keywords: spleen, splenectomy, sclerosing, angiomatoid nodular, transformation

\section{INTRODUCTION}

Sclerosing angiomatoid nodular transformation (SANT), a rare non-neoplastic vascular disease affecting the spleen, is formally known as hemangioma or multinodular hemangioma (1). Officially, SANT was first defined by Martel et al. after reviewing 25 cases from articles published before 2004 (2). It usually occurs in the middle-aged population (3) with a female pre-dominance (4). The vast majority of SANT patients are usually symptom free, with lesions detected occasionally by physical examinations (5) or during the treatment of other unrelated diseases (6). Some cases may present non-specific symptoms, such as abdominal pain (7), nausea, vomiting, and malnutrition (8). To date, the diagnosis of SANT is still a challenge without histological examinations, despite the fact that computerized tomography $(\mathrm{CT})$ and magnetic resonance imaging (MRI) provide 
imaging support before surgery $(5,6)$. In clinical practice, open or laparoscopic splenectomy is chosen for the treatment of SANT to avoid the risk of spontaneous rupture (9) and the suspicion of malignancy. In this study, we retrospectively analyzed the clinical and pathological features of five SANT cases admitted to our hospital between July 2010 and November 2019. Our study is a good complement for the whole SANT cohort.

\section{MATERIALS AND METHODS}

\section{Ethical Approval}

The study protocols were approved by the Ethics Committee of Shaoxing People's Hospital. Written informed consent was obtained from each patient for the publication of this article and any accompanying tables and/or images.

\section{Patients and Clinical Data}

We searched our pathological data and found diagnosed SANT cases based on pathological reports; then these reports were reviewed retrospectively. The clinical data of each patient were collected from the hospital records, including clinical materials and imaging results. Then the data were analyzed retrospectively by qualified staff experienced in the diagnosis of SANT. The postoperative complications were measured with the Clavien-Dindo classification (10).

\section{Imaging Analysis}

Two patients underwent ultrasonography (US) (Mindray, Resona $7 \mathrm{~T}, 5.0 \mathrm{mHz}$ ) in our hospital, and three other patients underwent US (parameters not available) in other hospitals. All the patients underwent a CT scan (GE, BrightSpeed 16/Phillips Brilliance 64) prior to surgery after US which indicated a mass in the spleen. Before examination, a fasting state for $8-10 \mathrm{~h}$ was necessary for each patient. The CT scan range was from the diaphragmatic surface to symphysis pubis, and the enhancement consisted of an arterial phase (30-35s), portal venous phase (60-65s), and delayed phase $(150 \mathrm{~s})$. US results were analyzed by two sonographers, and CT scans were assessed by three radiologists. All the decisions on the diagnosis were made upon consensus.

\section{Pathological Analysis}

All the specimens obtained after surgery were fixed with $4 \%$ neutral formalin and processed by dehydration and paraffin embedding. Then the sections $(3-4 \mu \mathrm{m})$ were subjected to hematoxylin-eosin (HE) staining, followed by observation under a light microscope to confirm the diagnosis of SANT. The immunohistochemical staining was performed by Envision plus. The sections were incubated with primary antibodies including CD34, CD31, CD8, and smooth muscle antibody (SMA) (Maixin Biotech, Fuzhou, China) and then with secondary antibodies plus a DAB chromogenic reagent (Dako). The pathological results were assessed by two pathologists blinded to this study. A detailed discussion was held until a consensus was reached in the presence of any disputes on the pathological reports.

Abbreviations: SANT, sclerosing angiomatoid nodular transformation; CT, computerized tomography; US, ultrasonography.
TABLE 1 | Pre-operative clinical data of SANT cases.

\begin{tabular}{llcll}
\hline Case & Gender & Age of onset & Symptoms & Signs \\
\hline 1 & Male & 39 & Incidentally found & Negative \\
2 & Female & 63 & Mild abdominal pain & Negative \\
3 & Female & 40 & Mild abdominal discomfort & Negative \\
4 & Male & 47 & Mild abdominal pain & Negative \\
5 & Male & 49 & Incidentally found & Negative \\
\hline
\end{tabular}

\section{RESULTS}

\section{Clinical Features}

Five SANT cases were found throughout our pathological data, including four cases initially confirmed and one case confirmed after pathological analysis in a retrospective way. Table 1 summarized the clinical data of five SANT patients (male: three; female: two). The mean age of onset was 47.6 years (39-63 years). Three patients presented with mild abdominal pain or discomfort in the left upper quadrant, while the other two patients were asymptomatic. The findings for physical examination were all normal for these patients. All denied a history of smoking, alcohol abuse, hypertension, diabetes, tumors, and other systemic diseases. Laboratory investigations including blood routine, biochemical analysis, and tumor markers were not remarkable in all cases.

\section{Imaging Findings}

Two cases underwent US in our hospital, which showed a hypoechoic or heterogeneous echotexture in the spleen. Meanwhile, color Doppler indicated internal vascularity in the lesion (Figure 1A). The imaging characteristics of the CT scans in the five cases were summarized in Table 2. All the SANT patients presented a solitary hypodense or complicated lesion area on the CT scan that was typically featured by marginal enhancement during the arterial phase (Figure 1B). The lesions were wellcircumscribed in a mean size of $3.94 \mathrm{~cm}(1.6-5.3 \mathrm{~cm})$, which were distributed in the superior pole $(n=1)$, middle pole $(n=2)$, and inferior pole $(n=2)$ of the spleen, respectively. Two cases showed a typical "spoke wheel" pattern (Figure 1B), and calcification in a lesion center was detected in one case (Figure 1C). No patient was diagnosed with SANT in our cohort pre-operatively.

\section{Surgical Data}

The intraoperative and post-operative data of these five cases are summarized in Table 3. Splenectomy was performed for all patients, with four cases under the laparoscopic approach and one case (spleen size, $13.2 \mathrm{~cm} \times 7.6 \mathrm{~cm} \times 8.4 \mathrm{~cm}$ ) under the open approach. The median operation time was $151 \mathrm{~min}$ (90-200 $\mathrm{min}$ ), and the intraoperative average blood loss was $160 \mathrm{ml} \mathrm{(50-}$ $300 \mathrm{ml}$ ). After surgery, all the patients showed thrombocythemia with a peak platelet count in a range of $391 \times 10^{9} / \mathrm{L}$ to 765 $\times 10^{9} / \mathrm{L}$ (Clavien-Dindo classification: Grade II). One patient presented with a Grade A pancreatic fistula (Clavien-Dindo classification: Grade II). These symptoms disappeared after oral administration of aspirin enteric-coated tablets (100 mg/day) for 

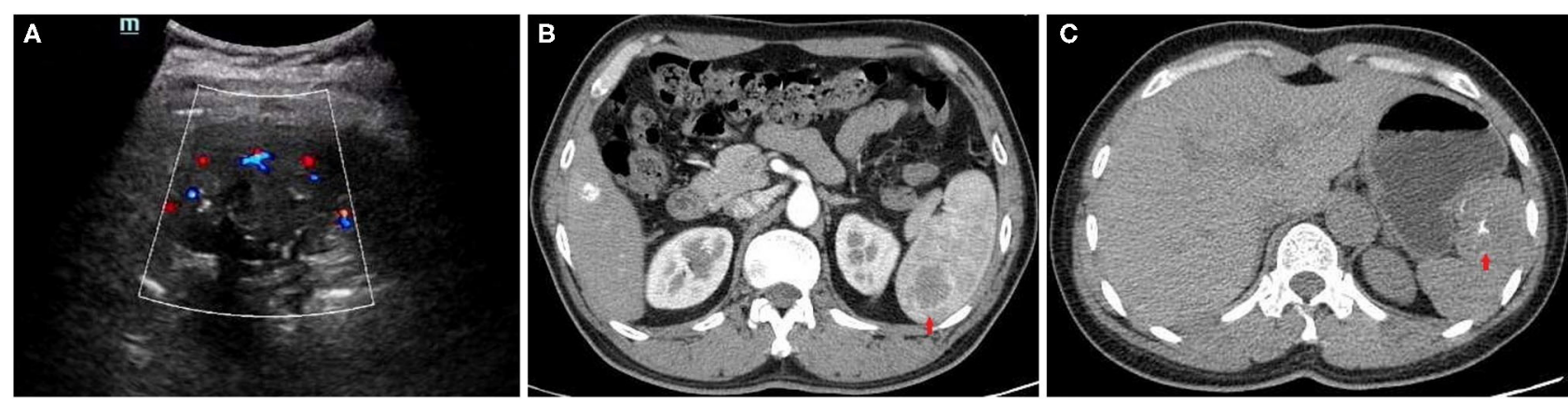

FIGURE 1 | Imaging findings of the SANT patients. (A) US showed a heterogeneous echotexture in the spleen, which was featured by internal vascularity on the color Doppler. (B) A "spoke wheel" pattern and peripheral enhancement around the SANT lesion was seen during the arterial phase. (C) A plain CT scan showed an iso-dense mass with central calcification in the spleen.

thrombocythemia and intravenous injection of somatostatin $(3$ $\mathrm{mg} /$ day) for the pancreatic fistula. No splenic vein thrombosis or other severe complications were detected. The mean hospital stay after surgery was 10 days (5-21 days). The median follow-up time was 40.5 months (8-116 months) with one patient lost in the follow-up, and no patient showed recurrence during this period.

\section{Pathological Characteristics}

Grossly, there was a solitary well-circumscribed mass in the splenic parenchyma in each case. The cut surface indicated that there were gray-white stellate scars in the central part, with reddish-brown nodules scattered peripherally. HE staining indicated multiple angiomatous nodules, which were wrapped by collagenized fibrous tissues in all lesions (Figure 2A). The angiomatous nodules were composed of slit-like vessels or sinusoids within which erythrocytes were diffused (Figure 2B). Obese endothelial cells lined the vessel lumens. Fusiform and ovoid cells were arranged in a target ring around the slit-like vessels (Figure 2B). The infiltration of lymphocytes and plasma cells could be seen in the stroma.

The immunohistochemical results displayed CD34(-) $\mathrm{CD} 31(+) \mathrm{CD} 8(+)$ for sinusoids, CD34(+) CD31(+) CD8(-) for capillaries, $\mathrm{CD} 34(-) \mathrm{CD} 31(+) \mathrm{CD} 8(-)$ for small veins (Figure 3), and $\operatorname{SMA}(+)$ for fusiform and ovoid cells.

\section{DISCUSSION}

SANT is a rare benign vascular disease in the red pulp of the spleen. Previously, there was no consensus on the nomenclature for this clinicopathological pattern. It was usually misdiagnosed as hemangioma, hemangiosarcoma, inflammatory pseudotumor, hamartoma (1), and even metastatic tumors (7). In 2004, Martel et al. summarized the pathological features and defined it as SANT for the first time (2). In line with our study, SANT usually affected the middle-aged population with an age of onset between 30 and 60 years (11). There was female predominance for SANT with a female-to-male ratio of 2:1 $(4,12)$; however, in our study, there was no female pre-dominance (three males and two females), which may be related to the limited sample size. Currently, the etiology of SANT is still unclear. In a previous study, Chang et al. speculated that the pathogenesis of SANT might be associated with vascular insufficiency and the subsequent vascular proliferation for repairing, indicating that SANT was just a polyclonal reactive lesion rather than genuine neoplasm (13). To our best knowledge, it is still a great challenge to carry out epidemiological studies for SANT as it shows a low morbidity.

The clinical manifestation of SANT is asymptomatic or non-specific, with most lesions identified incidentally (5) or during the treatment of other unrelated diseases (6). Others exhibited featureless symptoms such as abdominal pain (7), nausea, vomiting, and malnutrition (8). Recently, Pelizzo et al. reported a case of SANT with spontaneous mass rupture and intraperitoneal hemorrhage in a 9 week-old female infant (9). In our study, two patients were detected incidentally, and the other three had slight abdominal pain or discomfort, which was consistent with most SANT cases.

Given a lack of specific biomarkers for SANT, pre-operative imaging studies should be of relatively great importance. Although US can conveniently detect the heterogeneous echotexture in the spleen and the internal vascularity on color Doppler can provide some clues for SANT, definite diagnosis is still a challenge due to the various appearances of SANT in US images (14). In the present study, two patients underwent Doppler scans in our hospital, which indicated only an euangiotic mass in the spleen. For the CT scan, SANT usually presented a hypo- or iso-dense complex mass which was located in any site of the spleen just like in our cases (5). According to the literature, central calcification and a "spoke wheel" pattern were usually considered typical manifestations in CT images, but these signs were not available in all patients $(5,6,15)$. For instance, of the five cases in our study, only one case had central calcification, and two cases showed a "spoke wheel" pattern. However, all five patients presented peripheral enhancement around the SANT lesion during the arterial phase. Thus, this imaging phenomenon should be taken into consideration for the diagnosis of SANT based on CT scans. MRI had some advantages in displaying certain imaging features of SANT (6). As there were no patients who underwent this examination in our study, we could not hold a deep discussion about it. 
TABLE 2 | The imaging characteristics of CT scans in five SANT cases.

\begin{tabular}{|c|c|c|c|c|c|c|}
\hline Patients & Num & Site & Size $(\mathbf{c m})$ & $\begin{array}{l}\text { Enhancement } \\
\text { (arterial phase) }\end{array}$ & Spoke wheel & Central calcification \\
\hline 1 & 1 & Inferior pole & $5.3 \times 5.0$ & Peripheral & $\mathrm{N}$ & $\mathrm{N}$ \\
\hline 2 & 1 & Middle pole & $1.6 \times 1.5$ & Peripheral & $\mathrm{N}$ & $\mathrm{N}$ \\
\hline 3 & 1 & Superior pole & $5.0 \times 4.0$ & Peripheral & Y & Y \\
\hline 4 & 1 & Inferior pole & $3.5 \times 2.5$ & Peripheral & Y & $\mathrm{N}$ \\
\hline 5 & 1 & Middle pole & $4.3 \times 3.9$ & Peripheral & $N$ & $\mathrm{~N}$ \\
\hline
\end{tabular}

Num, number; cm, centimeter; Y, yes; N, no.

TABLE 3 | Intraoperative and post-operative data of five SANT cases.

\begin{tabular}{|c|c|c|c|c|c|c|}
\hline Patients & OP mode & $\begin{array}{l}\text { OP time } \\
\text { (min) }\end{array}$ & $\begin{array}{l}\text { Blood loss } \\
\text { (ml) }\end{array}$ & $\begin{array}{l}\text { Peak platelet count } \\
\qquad\left(10^{9} / \mathrm{L}\right)\end{array}$ & $\begin{array}{l}\text { Hospital stay } \\
\text { (days) }\end{array}$ & $\begin{array}{c}\text { Follow-up } \\
\text { (months) }\end{array}$ \\
\hline 1 & OS & 110 & 150 & 647 & 21 & 116 \\
\hline 2 & LS & 160 & 300 & 580 & 10 & Lost \\
\hline 3 & LS & 90 & 50 & 391 & 5 & 22 \\
\hline 4 & LS & 200 & 200 & 457 & 7 & 16 \\
\hline 5 & LS & 195 & 100 & 765 & 7 & 8 \\
\hline
\end{tabular}

OP, operation; OS, open splenectomy; LS, laparoscopic splenectomy; $\mathrm{ml}$, milliliter; L, liter.
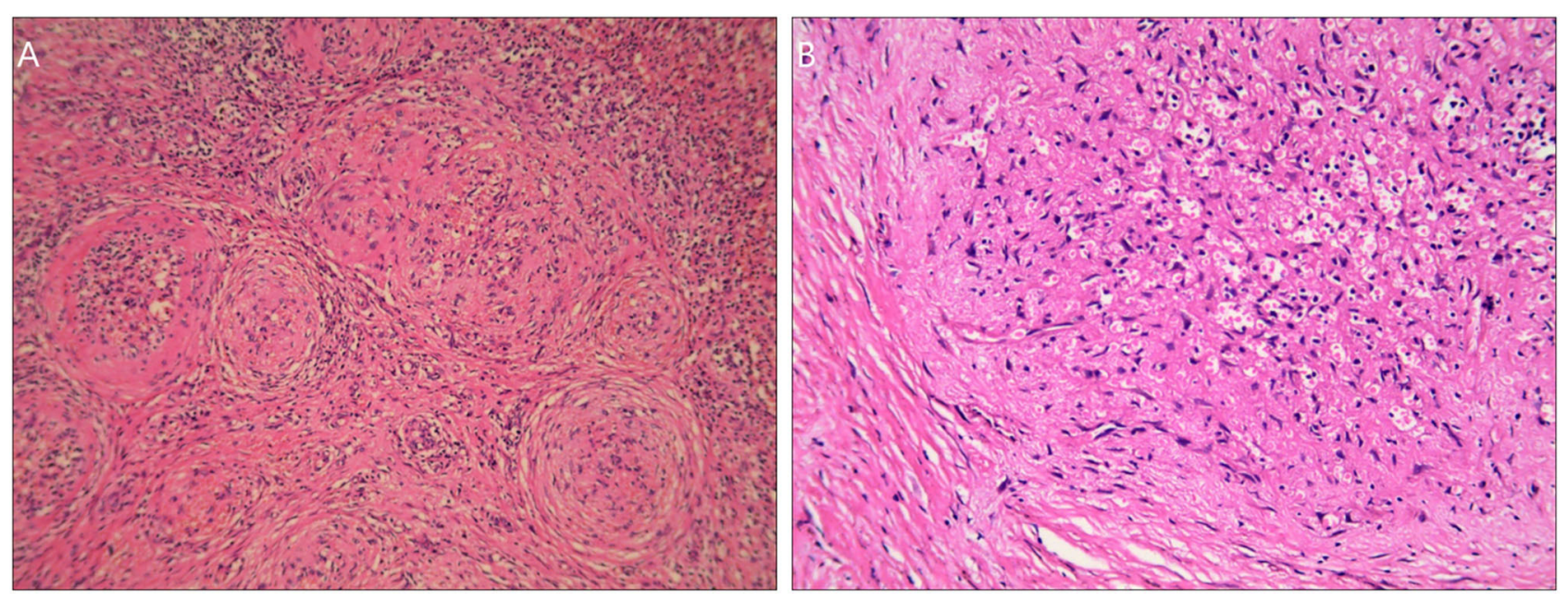

FIGURE 2 | HE staining images of the SANT patients. (A) Multiple angiomatous nodules with collagenized fibrous tissues in the peripheral part under a magnification of $<200 \times$. (B) There was an angiomatous nodule composed of slit-like vessels or sinusoids. Obese endothelial cells lined the vessel lumens. Fusiform and ovoid cells were arranged in a target ring round the nodule under a magnification of $200 \times$.

Generally, splenectomy is considered as the first-line option for SANT (12), avoiding the risk of spontaneous rupture (9) and the suspicion of malignancy. Open and laparoscopic approaches are alternative options depending on the different situations of the patients (16). For patients with a large spleen after a preoperative imaging assessment, such as the open case in this study, an open surgery is preferred as the large spleen may limit the laparoscopic space and may increase difficulties during the laparoscopic approach. However, for surgeons experienced in laparoscopy, a laparoscopic surgery can still be tried. In our study, there were no significant differences in the operation time, blood loss, and post-operative complications between patients treated by different surgical approaches. However, the hospital stay seemed to be shorter for patients who underwent the laparoscopic approach. These results should be further verified due to the limited number of cases. During the laparoscopic approach, there is a high possibility of splenic rupture. Nowadays, confusion still arises in the pre-operative diagnosis of SANT although it is a benign vascular lesion. Furthermore, we could not absolutely exclude the probability of malignancy. Thus, we propose that the preservation of spleen integrality is of great importance during surgery, in order to achieve en bloc 

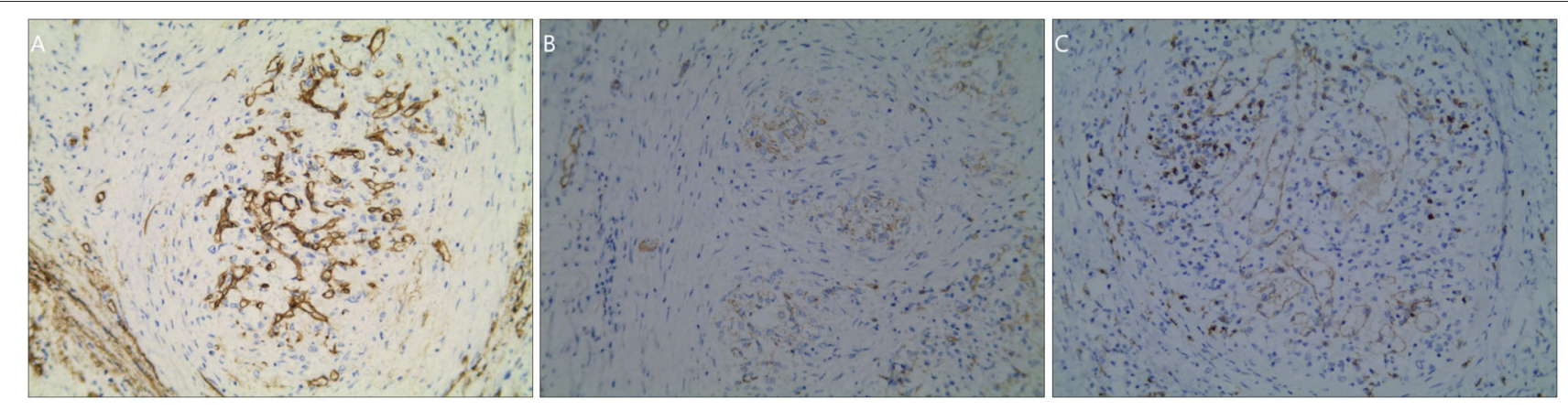

FIGURE 3 | The immunophenotype of the SANT patients under a magnification of 200x. (A) Immunohistochemical staining for CD34. (B) Immunohistochemical staining for CD31. (C) Immunohistochemical staining for CD8.

resections. To our best knowledge, the prognosis of SANT is favorable after surgical resections $(6,17)$ with no recurrence in previous literature.

To date, the pathological characteristics of SANT have been well-defined. Macroscopically, SANT usually presented as an isolated well-demarcated mass with a gray-white stellate scar in the central part. Besides, reddish-brown nodules were localized in the peripheral sites $(2,6)$. Multiple angiomatous nodules wrapped by collagenized fibrous tissues were the most typical traits for SANT microscopically. The nodules were usually composed of slit-like vessels or sinusoids and fibrous tissues constituted of fusiform and ovoid cells which were arranged in target ring around the nodules $(2,6,11)$. Erythrocytes could be seen in the vessel lumens, while the stroma was infiltrated by lymphocytes and plasma cells. All these features were well in line with our cases.

According to previous literature, the immunophenotype of SANT was almost similar among reported cases, which was featured by CD34(-) CD31(+) CD8(+) for sinusoids, CD34(+) CD31(+) CD8(-) for capillaries, and CD34(-) CD31(+) CD8(-) for small veins $(2,18,19)$. These three types of vessels were seemingly mimicking the structure of red pulp, which indicated that SANT may originate from red pulp.

\section{CONCLUSIONS}

SANT is a rare benign vascular entity affecting the spleen. A CT scan is relatively important for SANT diagnosis pre-operatively. Peripheral enhancement around the lesion during the arterial phase should be taken into consideration as an imaging sign on CT scans for SANT diagnosis. Open or laparoscopic splenectomy

\section{REFERENCES}

1. Krishnan J, Frizzera G. Two splenic lesions in need of clarification: hamartoma and inflammatory pseudotumor. Semin Diagn Pathol. (2003) 20:94-104. doi: 10.1016/S0740-2570(03)00014-5

2. Martel M, Cheuk W, Lombardi L, Lifschitz-Mercer B, Chan JK, Rosai J. Sclerosing angiomatoid nodular transformation (SANT): report of 25 cases is an alternative for SANT treatment. Preservation of spleen integrality should be preferred, especially during the laparoscopic approach, because of the probability of malignancy and the fragility of the spleen. According to pathological features, SANT may originate from the red pulp of the spleen.

\section{DATA AVAILABILITY STATEMENT}

The raw data supporting the conclusions of this article will be made available by the authors, without undue reservation.

\section{ETHICS STATEMENT}

The studies involving human participants were reviewed and approved by The Ethics Committee of Shaoxing People's Hospital. The patients/participants provided their written informed consent to participate in this study. Written informed consent was obtained from the individual(s) for the publication of any potentially identifiable images or data included in this article.

\section{AUTHOR CONTRIBUTIONS}

HS wrote the manuscript. FL revised the manuscript. BL completed the data analysis. ZS collected the data. All authors have read and approved the article.

\section{FUNDING}

The work was sponsored by the Science and Technology Planning Project of Shaoxing (No. 2018C30091).

3. Kuo TT, Chen TC, Lee LY. Sclerosing angiomatoid nodular transformation of the spleen (SANT): clinicopathological study of 10 cases with or without abdominal disseminated calcifying fibrous tumors, and the presence of a significant number of IgG4+ plasma cells. Pathol Int. (2009) 59:84450. doi: $10.1111 /$ j.1440-1827.2009.02456.x 
4. Li L, Fisher DA, Stanek AE. Sclerosing angiomatoid nodular transformation (SANT) of the spleen: addition of a case with focal CD68 staining and distinctive CT features. Am J Surg Pathol. (2005) 29:839-41. doi: 10.1097/01.pas.0000160441.23523.d1

5. Vigorito R, Scaramuzza D, Pellegrinelli A, Marchianò A. Sclerosing angiomatoid nodular transformation (SANT) of the spleen: a case report on CT and MRI. BJR Case Rep. (2019) 5:20180036. doi: 10.1259/bjrcr.20180036

6. Ma J, Zhang W, Wang L, Zhu Z, Wang J, Zhang J, et al. Imaging features of sclerosing angiomatoid nodular transformation in spleen. J Comput Assist Tomogr. (2019) 43:863-9. doi: 10.1097/RCT.0000000000000910

7. Pinheiro JL, Catarino S. Sclerosing angiomatoid nodular transformation of the spleen: case report of a metastatic carcinoma-simulating disorder. J Surg Case Rep. (2019) 2019:rjz249. doi: 10.1093/jscr/rjz249

8. Chikhladze S, Lederer AK, Fichtner-Feigl S, Wittel UA, Werner M, Aumann K. Sclerosing angiomatoid nodular transformation of the spleen, a rare cause for splenectomy: two case reports. World J Clin Cases. (2020) 8:1039. doi: 10.12998/wjcc.v8.i1.103

9. Pelizzo G, Villanacci V, Lorenzi L, Doria O, Caruso AM, Girgenti V, et al. Sclerosing angiomatoid nodular transformation presenting with abdominal hemorrhage: first report in infancy. Pediatr Rep. (2019) 11:7848. doi: 10.4081/pr.2019.7848

10. Clavien PA, Barkun J, de Oliveira ML, Vauthey JN, Dindo D, Schulick RD, et al. The Clavien-Dindo classification of surgical complications: five-year experience. Ann Surg. (2009) 250:187-96. doi: 10.1097/SLA.0b013e3181b13ca2

11. Falk GA, Nooli NP, Morris-Stiff G, Plesec TP, Rosenblatt S. Sclerosing angiomatoid nodular transformation (SANT) of the spleen: case report and review of the literature. Int J Surg Case Rep. (2012) 3:492500. doi: 10.1016/j.ijscr.2012.06.003

12. Cipolla C, Florena AM, Ferrara G, Di Gregorio R, Unti E, Giannone AG, et al. Sclerosing angiomatoid nodular transformation: laparoscopic splenectomy as therapeutic and diagnostic approach at the same time. Case Rep Surg. (2018) 2018:7020538. doi: 10.1155/2018/7020538
13. Chang KC, Lee JC, Wang YC, Hung LY, Huang Y, Huang WT, et al. Polyclonality in sclerosing angiomatoid nodular transformation of the spleen. Am J Surg Pathol. (2016) 40:1343-51. doi: 10.1097/PAS.0000000000000716

14. Wang HL, Li KW, Wang J. Sclerosing angiomatoid nodular transformation of the spleen: report of five cases and review of literature. Chin Med J. (2012) 125:2386-9.

15. Karaosmanoglu DA, Karcaaltincaba M, Akata D. CT and MRI findings of sclerosing angiomatoid nodular transformation of the spleen: spoke wheel pattern. Korean J Radiol. (2008) 9:S52-5. doi: 10.3348/kjr.2008.9.s.s52

16. Jin $\mathrm{Y}, \mathrm{Hu} \mathrm{H}$, Regmi P, Li F, Cheng N. Treatment options for sclerosing angiomatoid nodular transformation of spleen. HPB. (2020) 22:157782. doi: 10.1016/j.hpb.2020.01.014

17. Koyama R, Minagawa N, Maeda Y, Shinohara T, Hamada T. A sclerosing angiomatoid nodular transformation (SANT) mimicking a metachronous splenic metastasis from endometrioid cancer and ovarian cancer. Int J Surg Case Rep. (2019) 65:292-5. doi: 10.1016/j.ijscr.2019.11.006

18. Bamboat ZM, Masiakos PT. Sclerosing angiomatoid nodular transformation of the spleen in an adolescent with chronic abdominal pain. J Pediatr Surg. (2010) 45:E13-6. doi: 10.1016/j.jpedsurg.2010.04.020

19. Pradhan D, Mohanty SK. Sclerosing angiomatoid nodular transformation of the spleen. Arch Pathol Lab Med. (2013) 137:1309-12. doi: 10.5858/arpa.2012-0601-RS

Conflict of Interest: The authors declare that the research was conducted in the absence of any commercial or financial relationships that could be construed as a potential conflict of interest.

Copyright (C) 2021 Shao, Lu, Shen and Liu. This is an open-access article distributed under the terms of the Creative Commons Attribution License (CC BY). The use, distribution or reproduction in other forums is permitted, provided the original author(s) and the copyright owner(s) are credited and that the original publication in this journal is cited, in accordance with accepted academic practice. No use, distribution or reproduction is permitted which does not comply with these terms. 